\title{
Effectiveness of Transarterial Chemoembolization- First Treatment for Advanced Hepatocellular Carcinoma: A Propensity Score Matching Analysis
}

\author{
Sung Won Chung $\mathbb{D}^{1, *}$ \\ Min Kyung Park (D) ${ }^{1, *}$ \\ Young Youn Cho $\mathbb{D}^{1,2, *}$ \\ Youngsu Park' \\ Cheol-Hyung Lee $\mathbb{D}^{\prime}$ \\ Hyunwoo Oh (iD) \\ Heejoon Jang (D) \\ Minseok Albert Kim (D) \\ Sun Woong Kim (D) \\ Joon Yeul Nam (D) \\ Yun Bin Lee $\mathbb{D}^{\prime}$ \\ Eun Ju Cho $\mathbb{D}^{\prime}$ \\ Su Jong $\mathrm{Yu} \mathbb{D}^{\prime}$ \\ Hyo-Cheol Kim (D) ${ }^{3}$ \\ Yoon Jun Kim (1D) \\ Jin Wook Chung (iD ${ }^{3}$ \\ Jung-Hwan Yoon (1D) \\ Jeong-Hoon Lee $\mathbb{I D}^{\prime}$
}

'Department of Internal Medicine and Liver Research Institute, Seoul National University College of Medicine, Seoul, Korea; ${ }^{2}$ Department of Internal Medicine, Chung-Ang University Hospital, Seoul, Korea; ${ }^{3}$ Department of Radiology, Seoul National University College of Medicine, Seoul, Korea

*These authors contributed equally to this work

Correspondence: Jeong-Hoon Lee Department of Internal Medicine and Liver Research Institute, Seoul National University College of Medicine, I0I

Daehak-Ro, Jongno-Gu, Seoul, 03080, Korea

Tel +82-2-2072-2228

Fax +82-2-743-670I

Email pindra@empal.com
Background: Still in real-world practice, advanced hepatocellular carcinoma (HCC) patients are treated with transarterial chemoembolization (TACE). This study compared the therapeutic effectiveness of initial TACE treatment and initial sorafenib treatment in advanced HCC patients.

Patient and Methods: Advanced HCC patients initially treated with sorafenib or TACE were included in this study. Treatment crossover due to an unfavorable response to initial treatment was allowed. Propensity score (PS) matching was applied for balancing baseline characteristics. The primary outcome was overall survival (OS) and the secondary outcomes included tumor response.

Results: A total of 554 patients were included in this study: 85 were initially treated with sorafenib (the sorafenib-first group) and 469 with TACE (the TACE-first group). In the entire cohort, the TACE-first group was associated with lower risk of death [adjusted hazard ratio $(\mathrm{HR})=0.75, P=0.04]$. In the PS-matched cohort ( 85 patients per group), the TACE-first group showed longer OS than the sorafenib-first group in both univariable $(\mathrm{HR}=0.68, P=0.02)$ and multivariable analyses (adjusted $\mathrm{HR}=0.58, P=0.002$ ). Specifically, within both the entire and the PS-matched cohorts, the TACE-first group showed longer OS in subgroups with major portal vein tumor thrombosis $(\mathrm{HR}=0.72, P=0.048$; $\mathrm{HR}=0.52, P=0.003)$ or infiltrative HCC (HR=0.42, $P<0.001 ; \mathrm{HR}=0.30, P=0.004$, respectively). The objective response rate was higher in the TACE-first group $(29.3 \%$ vs $14.7 \%, P=0.03)$ within the PS-matched cohort.

Conclusion: For advanced HCC, initial TACE leads to longer OS with a more favorable tumor response than initial sorafenib treatment. Intrahepatic tumor control with initial locoregional therapy may be a potent strategy for advanced HCC.

Keywords: liver cancer, transarterial therapy, locoregional therapy, tyrosine kinase inhibitor

\section{Introduction}

Liver cancer is one of the most common cancers worldwide. It is the second most common cause of cancer-related deaths in men and the sixth most common in women. ${ }^{1}$ As hepatocellular carcinoma (HCC) accounts for $90 \%$ of primary liver cancers, it constitutes a major global health burden. Unfortunately, more than half of HCCs are detected at an advanced stage. ${ }^{2}$

For advanced HCC [Barcelona Clinic Liver Cancer (BCLC) stage C], sorafenib had been recommended in international guidelines as the sole standard therapy before the introduction of lenvatinib and bevacizumab plus atezolizumab ${ }^{3,4}$ since $^{2}$ sorafenib is the first treatment proven to prolong overall survival (OS) in patients 
with advanced HCC. ${ }^{5,6}$ There had been no proven secondline treatment in advanced $\mathrm{HCC}$ before the introduction of regorafenib, ${ }^{7}$ cabozantinib, ${ }^{8}$ ramucirumab, ${ }^{9}$ nivolumab,${ }^{10}$ and pembrolizumab. ${ }^{11}$ In most advanced HCC, patients die of liver failure due to intrahepatic tumor progression. ${ }^{12}$ In another study, advanced HCC patient with extrahepatic metastasis, intrahepatic tumor control was positively associated with prolonged OS. ${ }^{13}$ These data support that intrahepatic tumor control could be the prime concern for advanced HCC patients. As locoregional treatment obliterates intrahepatic tumors rapidly, it could play some role in advanced HCC. In this regard, transarterial chemoembolization (TACE) had been a potential salvage treatment option among the locoregional treatments. Even though the current guidelines do not recommend TACE in patients with extrahepatic metastasis or portal vein tumor thrombosis (PVTT), ${ }^{4,14}$ several studies have shown promising results in expanding TACE indications. ${ }^{15,16}$ Moreover, a recent randomized control trial showed that combination therapy of TACE and radiation achieved both progression delay and survival benefits compared with sorafenib alone in advanced HCC patients with major vascular invasion. ${ }^{17} \mathrm{TACE}$ had played a role in treating advanced HCC in sorafenib failure patients or as the first-line treatment even before sorafenib treatment in real-world practice. ${ }^{2}$

Unfortunately, the impact of the sequence of a systemic treatment represented by sorafenib and TACE was not thoroughly evaluated. Therefore, in this study, we compared OS and tumor response between sorafenib-first treatment and TACE-first treatment for advanced HCC.

\section{Patients and Methods}

This was a retrospective analysis of prospectively established cohort. The prospective cohort included consecutive patients with advanced HCC at a single tertiary referral center (Seoul National University Hospital, Seoul, South Korea) from July 2005. Among the prospective cohort, patients who were diagnosed with advanced HCC from November 2006 to October 2015 were analysed in this study. Advanced HCC was defined in accordance with the BCLC staging system: HCC with either macrovascular invasion or extrahepatic spread (lymph node involvement or metastasis). ${ }^{14}$ Eligible patients required a Child-Pugh class of A or B and Eastern Cooperative Oncology Group performance status of 2 or less. Patients were also eligible if they were unsuitable for hepatic resection or liver transplantation. Patients were excluded if they met any of the following criteria: prior diagnosis of malignancies except for HCC within 5 years; prior or current treatment with systemic cytotoxic chemotherapy; other severe systematic comorbidities such as end-stage renal disease, advanced heart failure, or chronic obstructive lung disease; and initial presentation as tumor rupture.

Baseline information such as demographic profile, laboratory findings, and liver disease aetiology was obtained at the time of advanced HCC diagnosis. PVTT was classified in accordance with the classification system of the Liver Cancer Study Group of Japan, ${ }^{18}$ and major PVTT was defined as tumor involvement of the first-order branches of the portal vein (ie, right and left portal vein, $\mathrm{Vp} 3)$ or the main portal vein (Vp4). Minor PVTT was defined as tumor involvement distal to, but not in, the second-order branches of the portal vein $(\mathrm{Vp} 1)$ or the second-order branches of the portal vein $(\mathrm{Vp} 2){ }^{18}$

This study was conducted in accordance with the declaration of Helsinki and approved by the institutional review board of Seoul National University Hospital (IRB No.: 1909-108-10) and all included patients provided informed consent to participate in our prospective cohort before enrolment (IRB No.: 0506-150-005).

\section{Treatment}

Patients were classified into either the sorafenib-first group (patients who underwent sorafenib as an initial treatment) or the TACE-first group (patients who underwent conventional TACE as an initial treatment). Sorafenib was administered orally at a dose of $400 \mathrm{mg}$ twice daily. Dose reduction or discontinuation of sorafenib was judged based on the manufacturer's instructions. Considering patient's residual liver function, patients' body weight, tumor volume, and feeding arteries (including collateral vessels), the conventional TACE was performed as selectively as possible using a microcatheter by experienced operators. Main TACE operators (JWC and HCK) were highly experienced interventional radiologists who have more than 28 years and 15 years of experience in TACE treatment, respectively. During the TACE procedure, meticulous tumor feeding artery searching was done (especially in patients with major PVTT). From December 2007, cone-beam computed tomography (CT) was utilized to detect tumor feeding artery in most cases. The caudate artery was selectively treated because major PVTT is commonly supplied by the caudate artery. ${ }^{19}$ An emulsion of iodized oil (2-12 $\mathrm{mL})$ and doxorubicin hydrochloride (10-60 mg) was infused into the tumor feeding 
artery until dense accumulation of iodized oil in the tumor was noted on fluoroscopy. The amount of emulsion administered was selected based on the tumor burden in each tumor-feeding artery. After the administration of the emulsion, absorbable gelatin sponge particles were administered to achieve near-stasis of target vessels (contrast media column does not clear-up within 5 heartbeats). Additional cisplatin infusion was performed at each operator's discretion if there was PVTT. ${ }^{20}$ Cisplatin was administered as a solution through tumor-feeding vessels at a concentration of $0.5 \mathrm{mg} / \mathrm{mL}$ and infused at the rate of 4-10 $\mathrm{mL} / \mathrm{min}$ after near-stasis was achieved. The total amount of cisplatin administered ranged from 50 to $100 \mathrm{mg}$ which was decided by the operator. Underlying patient's liver function, tumor volume, and patient's body weight were factors in deciding cisplatin dose. In patients with mild arterioportal shunts, alternative injection of iodized oil emulsion and gelatin sponge articles was commonly performed to minimize leakage of iodized oil emulsion into the portal vein and maximize accumulation of iodized oil in the tumor. In patients with severe arterioportal shunts, embolization with absorbable gelatin sponge particles was performed to occlude the shunting, and then additional cisplatin infusion was undertaken. TACE was performed on demand if there was a residual or recurrent tumor. However, cases of transarterial radioembolization or drug-eluting bead TACE were excluded.

Subsequent treatment crossover (eg, TACE or another locoregional treatment following sorafenib in the sorafenib-first group and sorafenib following TACE in the TACE-first group) was allowed. The sorafenib-first group underwent TACE upon documentation of progressive disease (PD) of the intrahepatic tumor in accordance with the modified Response Evaluation Criteria in Solid Tumors (mRECIST) guidelines with no contraindication for TACE (ie, decompensated cirrhosis such as hepatic encephalopathy, Child-Pugh score $\geq 10$ points, intractable ascites, or variceal bleeding). ${ }^{14}$ The TACE-first group was treated with sorafenib either upon the progression of additional extrahepatic metastasis, including a non-target lesion, in accordance with mRECIST, ${ }^{21}$ or when two consecutive sessions of TACE were ineffective, which was confirmed by multiphase abdomen-pelvis $\mathrm{CT}$ or magnetic resonance imaging (MRI). ${ }^{22}$

A newly developed single lesion could be treated by other locoregional therapy such as percutaneous ethanol injection, radiofrequency ablation, radiotherapy, or metastatectomy. The locoregional therapy selection was at the physician's discretion.

\section{Outcomes and Evaluation}

The primary outcome was OS and the secondary outcomes were TTP and tumor response. The index date was set as the date when advanced HCC was diagnosed. OS was defined as the time from the index date to the date of death from any cause. TTP was measured from the index date until the date of earliest disease progression. Patients who died before radiologic tumor response evaluation were excluded from the TTP analysis.

HCC tumor response was evaluated by the best overall response (BOR). Contrast-enhanced abdomen-pelvis dynamic CT and MRI using liver-specific contrast agents were used for the measurement of tumor response. The radiologic tumor evaluation was conducted at baseline and every 1-3 months during treatment unless the patient showed a sudden clinical deterioration. BOR was identified in accordance with mRECIST criteria. ${ }^{21}$ BOR was defined as the best tumor response throughout the treatment period. The objective response rate (ORR) was defined as the proportion of patients with a complete response $(\mathrm{CR})$ or partial response (PR). The ORR were evaluated with BOR. Median duration of response was measured among patients who achieved PR and CR. ${ }^{23}$

\section{Statistical Analysis}

The following factors were used for propensity-score (PS) stratification: 1) the presence of metastasis, 2) age, 3) the presence of cirrhosis, 4) tumor type (ie, infiltrative HCC vs nodular HCC), 5) maximal diameter of intrahepatic tumor, 6) baseline creatinine level, 7) the presence of PVTT, 8) Child-Pugh class, and 9) alpha-fetoprotein (AFP) level. Nearest-neighbour matching was applied for PS matching.

Categorical baseline characteristics were analysed using chi-squared test or Fisher's exact test. Continuous variables were evaluated with Student's $t$-test. BOR and ORR were compared using chi-squared exact test. OS and TTP were estimated by the Kaplan-Meier curves and were compared using the Log rank test. Multivariable Cox proportional regression analysis was performed to compute the hazard ratio (HR).

All analyses were performed using $\mathrm{R}$ language version 4.00 ( $\mathrm{R}$ Foundation for Statistical Computing, Vienna, Austria). A $P$-value less than 0.05 was considered statistically significant. 


\section{Results}

\section{Baseline Characteristics}

During the study period, 554 patients were included in our analysis (Supplementary Figure S1). In addition, a PSmatched cohort consisting of 170 patients (at a 1:1 ratio, 85 patients per group) was established. Before PS matching, there were significant differences in patient age, infiltrative tumor type, PVTT at diagnosis, maximum diameter of the intrahepatic tumor, presence of extrahepatic metastasis (lymph node or visceral metastasis), baseline serum creatinine, and AFP (Table 1). There was no significant difference between the two treatment groups regarding patients who were treated with another modality such as radiotherapy or radiofrequency ablation (the TACE-first group vs the sorafenib-first group: $20.3 \%$ vs $15.3 \%$, $P=0.37$ by Fisher's exact test). After PS matching, there were no significant differences between the two treatment groups (Table 1) and they were generally well balanced (Table 1). Patients who underwent treatment crossover tend to have significant survival benefit compared to the patients who did not. This result was consistent in both entire-cohort (treatment crossover vs no treatment crossover: $\mathrm{HR}=0.68 ; 95 \% \mathrm{CI}=0.55-0.83 ; P<0.001)$ and PSmatched cohort $(\mathrm{HR}=0.56 ; 95 \% \mathrm{CI}=0.40-0.78 ; P<0.001)$.

\section{Overall Survival and Time to Progression of the Entire Cohort}

The data cut-off date for both OS and TTP analyses was May 20, 2020. In the entire cohort, there was no significant difference in OS in univariable analysis (the TACEfirst group vs the sorafenib-first group: $\mathrm{HR}=0.91,95 \%$ confidence interval $(\mathrm{CI})=0.71-1.17, P=0.47$ by Log rank test; Supplementary Figure S2A). However, the TACEfirst group was independently associated with reduced risk of death in multivariable analysis (adjusted HR $(\mathrm{aHR})=0.75 ; 95 \% \mathrm{CI}=0.57-0.99 ; P=0.04$; Table 2 ) after adjustment for age, maximal intrahepatic tumor diameter, AFP level, infiltrative tumor type, presence of visceral metastasis, presence of major PVTT (Vp3 or Vp4), presence of underlying cirrhosis, and Child-Pugh class. There was no significant TTP difference between the two treatment groups (Supplementary Figure S2B).

\section{Overall Survival and Time to Progression of the PS-Matched Cohort}

In the PS-matched cohort, the TACE-first group showed a significantly lower risk of death than the sorafenib-first group $(\mathrm{HR}=0.68,95 \% \mathrm{CI}=0.49-0.95, P=0.02$ by $\log$ rank test; Figure 1A). The median OS was 10.9 months $(95 \%$ $\mathrm{CI}=8.8-14.4$ months) in the TACE-first group and 6.7 months $(95 \% \mathrm{CI}=5.2-8.1$ months) in the sorafenib-first group. The multivariable analysis also showed a consistent survival benefit in the TACE-first group compared with the sorafenib-first group $(\mathrm{aHR}=0.58,95 \%$ $\mathrm{CI}=0.41-0.82, P=0.002$; Table 2) within the PS-matched cohort.

After matching, there was still no significant difference in TTP between the two treatment groups (the TACE-first group vs the sorafenib-first group: $\mathrm{HR}=1.01,95 \%$ $\mathrm{CI}=0.73-1.42, P=0.92$ by Log rank test; Figure 1B) after matching. The ORR $(\mathrm{CR}+\mathrm{PR})$ was significantly higher in the TACE-first group than in the sorafenib-first group ( $29.3 \%$ vs $14.7 \%, P=0.03$ by chi-squared test; Table 3 ).

To further evaluate the discrepancy between OS and TTP within the PS-matched cohort, patients were divided by their objective response and subgroup analysis was performed. Among patients who achieved objective responses (ie, PR or $\mathrm{CR}$ ), the sorafenib-first group showed significantly longer TTP $(\mathrm{HR}=3.96 ; 95 \% \mathrm{CI}=1.46-10.71, P=0.004$ by $\log$ rank test; Figure 2A). The median duration of response was 11.0 months ( $95 \% \mathrm{CI}=9.1$ months - not reached) in the sorafenibfirst group and 5.0 months ( $95 \% \mathrm{CI}=2.9-8.3$ months) in the TACE-first group. However, there was no significant difference in OS between the two groups $(\mathrm{HR}=2.10 ; 95 \%$ $\mathrm{CI}=0.82-5.38, P=0.12$ by Log rank test; Figure $2 \mathrm{~B}$ ). On the other hand, within patients who failed to achieve PR or CR (ie, stable disease or PD), there was no significant difference in TTP $(\mathrm{HR}=0.82 ; 95 \% \mathrm{CI}=0.56-1.19, P=0.29$ by Log rank test; Figure 2C), but the TACE-first group showed longer OS (HR $=0.61,95 \% \mathrm{CI}=0.41-0.89, P=0.01$ by Log rank test; Figure 2D).

\section{Subgroup Analysis of Overall Survival}

We further divided the patients according to treatment crossovers, $\mathrm{HCC}$ characteristics, and underlying liver function. In the entire cohort, in patients with infiltrative $\mathrm{HCC}(\mathrm{HR}=0.42$, $95 \% \mathrm{CI}=0.26-0.69, P<0.001$ by Log rank test) and major PVTT $(\mathrm{HR}=0.72,95 \% \mathrm{CI}=0.52-0.99, P=0.048$ by Log rank test), the TACE-first group showed a survival benefit compared with the sorafenib-first group (Figure 3A). Similar results were reproduced in PS-matched patients (Figure 3B). The TACE-first group showed a significant survival benefit over the sorafenib-first group in subgroup of patients with major PVTT $(\mathrm{HR}=0.52,95 \% \mathrm{CI}=0.34-0.80, P=0.003$ by Log rank test), no treatment crossover ( $\mathrm{HR}=0.57,95 \%$ 
Table I Baseline Characteristics and Demographics of the Patients

\begin{tabular}{|c|c|c|c|c|c|c|c|}
\hline \multirow[t]{2}{*}{ Characteristics } & \multicolumn{3}{|c|}{ Entire Cohort $(n=554)$} & \multicolumn{4}{|c|}{ PS-Matched Cohort $(n=170)$} \\
\hline & $\begin{array}{l}\text { Sorafenib-First } \\
\qquad(n=85)\end{array}$ & $\begin{array}{l}\text { TACE-First } \\
(n=469)\end{array}$ & $P$ value & $\begin{array}{c}\text { Sorafenib-First } \\
\qquad(n=85)\end{array}$ & $\begin{array}{l}\text { TACE-First } \\
\qquad(n=85)\end{array}$ & $P$ value & SMD \\
\hline Age, years & $59(53-67)$ & $56(49-64)$ & 0.02 & $59(53-67)$ & $58(54-66)$ & 0.78 & 0.04 \\
\hline Male Sex, N (\%) & 71 (83.5\%) & $398(84.9 \%)$ & 0.88 & 71 (83.5\%) & 67 (78.8\%) & 0.56 & 0.12 \\
\hline \multicolumn{8}{|l|}{ Etiology, N (\%) } \\
\hline HBV & $65(76.5 \%)$ & $330(70.4 \%)$ & 0.30 & 65 (76.5\%) & 73 (85.9\%) & 0.17 & 0.24 \\
\hline $\mathrm{HCV}$ & $6(7.1 \%)$ & $23(4.9 \%)$ & 0.43 & $6(7.1 \%)$ & $4(4.7 \%)$ & 0.75 & 0.10 \\
\hline Alcoholic & $5(5.9 \%)$ & $45(9.6 \%)$ & 0.41 & $5(5.9 \%)$ & $6(7.1 \%)$ & 1.00 & 0.05 \\
\hline Non-viral non-alcoholic & $9(10.6 \%)$ & 90 (19.1\%) & 0.06 & $9(10.6 \%)$ & 7 (8.2\%) & 0.79 & 0.08 \\
\hline Underlying cirrhosis, $\mathrm{N}(\%)$ & $82(96.5 \%)$ & $432(92.1 \%)$ & 0.23 & $82(96.5 \%)$ & $84(98.8 \%)$ & 0.62 & 0.16 \\
\hline \multicolumn{8}{|l|}{ HCC characteristics } \\
\hline Infiltrative HCC, N (\%) & 19 (22.4\%) & $269(57.4 \%)$ & $<0.001$ & $19(22.4 \%)$ & $15(17.6 \%)$ & 0.57 & 0.12 \\
\hline PVTT at diagnosis, N (\%) & & & $<0.001$ & & & 0.78 & 0.16 \\
\hline$V_{p} 0$ or $V_{p} I$ & $30(35.3 \%)$ & 57 (12.2\%) & & $30(35.3 \%)$ & $31(36.5 \%)$ & & \\
\hline$v_{p 2}$ & $5(5.9 \%)$ & 96 (20.5\%) & & $5(5.9 \%)$ & $6(7.1 \%)$ & & \\
\hline$V_{p} 3$ & $20(23.5 \%)$ & $125(26.7 \%)$ & & $20(23.5 \%)$ & $24(28.2 \%)$ & & \\
\hline$V_{p} 4$ & 30 (35.3\%) & 191 (40.7\%) & & 30 (35.3\%) & $24(28.2 \%)$ & & \\
\hline $\begin{array}{l}\text { Major } \mathrm{PVTT}^{\dagger} \text { at diagnosis, } \\
\mathrm{N}(\%)\end{array}$ & $50(58.8 \%)$ & $316(67.4 \%)$ & 0.15 & $50(58.8 \%)$ & $48(56.5 \%)$ & 0.88 & 0.05 \\
\hline HCC maximum diameter, cm & $5.5(2.0-10.0)$ & $9.5(5.7-12.1)$ & $<0.001$ & $5.5(2.0-10.0)$ & $6.1(3.9-10.0)$ & 0.77 & 0.04 \\
\hline Extrahepatic Metastasis, N (\%) & $55(64.7 \%)$ & 98 (20.9\%) & $<0.001$ & $55(64.7 \%)$ & $54(63.5 \%)$ & 1.00 & 0.03 \\
\hline Bone meta, N (\%) & $6(7.1 \%)$ & $18(3.8 \%)$ & 0.24 & $6(7.1 \%)$ & II (I2.9\%) & 0.31 & 0.20 \\
\hline LN meta, $\mathrm{N}(\%)$ & $22(25.9 \%)$ & $32(6.8 \%)$ & $<0.001$ & $22(25.9 \%)$ & $20(23.5 \%)$ & 0.86 & 0.06 \\
\hline Visceral meta, $\mathrm{N}(\%)$ & $28(32.9 \%)$ & $63(13.4 \%)$ & $<0.001$ & $28(32.9 \%)$ & 30 (35.3\%) & 0.87 & 0.05 \\
\hline Child-Pugh class, N (\%) & & & & & & 1.00 & $<0.001$ \\
\hline Class A, N (\%) & $68(80.0 \%)$ & $326(69.5 \%)$ & & 69 (80.0\%) & 69 (80.0\%) & & \\
\hline Class B, N (\%) & 17 (20.0\%) & 143 (30.5\%) & 0.05 & $17(20.0 \%)$ & 17 (20.0\%) & & \\
\hline \multicolumn{8}{|l|}{ Laboratory data } \\
\hline AFP, $n g / m L$ & $429(13-6790)$ & II $90(58-33800)$ & $<0.001$ & $429(13-6790)$ & $258(16-5090)$ & 0.06 & 0.29 \\
\hline ALT, IU/L & $43(26-69)$ & $47(31-74)$ & 0.08 & $43(26-69)$ & $43(27-73)$ & 0.27 & 0.17 \\
\hline Total bilirubin, $\mathrm{mg} / \mathrm{dL}$ & I.I (0.9-1.6) & I.I (0.8-I.6) & 0.71 & I.I (0.9-I.6) & $0.9(0.7-1.4)$ & 0.18 & 0.21 \\
\hline Hemoglobin, g/dL & $13.1(\mid 1.8-14.1)$ & $13.1(\mid 1.9-14.3)$ & 0.17 & $13.1(\mid 1.8-14.1)$ & $12.8(\mid 1.6-14.2)$ & 0.81 & 0.04 \\
\hline $\mathrm{Cr}, \mathrm{mg} / \mathrm{dL}$ & $0.83(0.73-0.98)$ & $0.9(0.79-1.00)$ & $<0.001$ & $0.83(0.73-0.98)$ & $0.85(0.77-0.99)$ & 1.00 & 0.001 \\
\hline
\end{tabular}

Notes: Data are expressed as N (\%) or median (interquartile range). PVTT was classified in accordance with the classification system of the Liver Cancer Study Group of Japan. ${ }^{\dagger}$ Major PVTT was defined as tumor involvement of the right or left portal vein $\left(V_{p} 3\right)$ or the main portal vein $\left(V_{p} 4\right)$.

Abbreviations: AFP, alpha-fetoprotein; ALT, alanine transferase; Cl, confidence interval; $\mathrm{Cr}$, creatinine; HBV, hepatitis B virus; HCC, hepatocellular carcinoma; HCV, hepatitis C virus; HR, hazard ratio; IQR, interquartile range; LN, lymph node; PVTT, portal vein tumor thrombosis; SMD, standardized mean difference; TACE, transarterial chemoembolization.

$\mathrm{CI}=0.35-0.94, P=0.03$ by $\log$ rank test), infiltrative $\mathrm{HCC}$ $(\mathrm{HR}=0.30,95 \% \mathrm{CI}=0.13-0.69, P=0.004$ by Log rank test $)$, Child-Pugh Class B (HR=0.40, 95\% CI=0.18-0.89, $P=0.02$ by Log rank test), or pretreatment serum AFP $\geq 200 \mathrm{ng} / \mathrm{mL}$ ( $\mathrm{HR}=0.51,95 \% \mathrm{CI}=0.33-0.80, \mathrm{P}=0.003$ by Log rank test). In patients with bone metastasis, the sorafenib-first group tended to have a survival benefit in both cohorts, but differences were not significant (entire cohort: HR=1.49, 95\%
$\mathrm{CI}=0.49-4.55, P=0.48$; PS-matched cohort: $\mathrm{HR}=1.34,95 \%$ $\mathrm{CI}=0.40-4.50, P=0.64)$.

\section{Discussion}

Our study showed that initial TACE treatment in patients with advanced HCC conferred a significant survival benefit compared with the sorafenib-first group in both the entire and the PS-matched cohorts. Although there was 


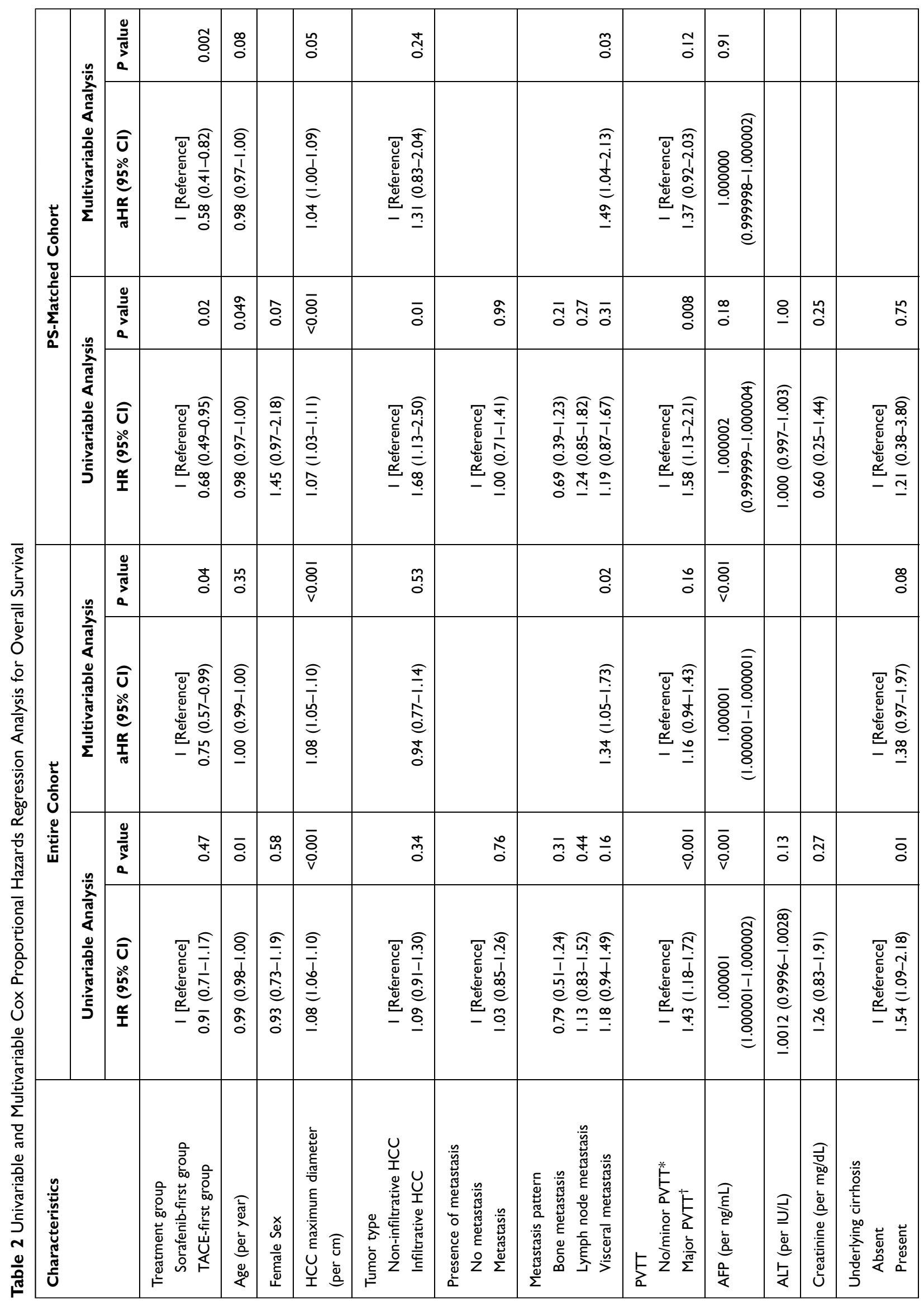


no difference in TTP between the two groups, ORR was significantly higher in the TACE-first group, which might explain the longer OS in that group. Subgroup analysis of the entire cohort showed that patients with more aggressive tumors, as defined by either the presence of major PVTT or infiltrative tumor type, had a survival benefit when they were initially treated with TACE. Similar results were reproduced in the PSmatched cohort: the presence of major PVTT, infiltrative tumor type, no treatment crossover group, high pretreatment AFP, and Child-Pugh class B were associated with survival benefit upon initial treatment with TACE.

There are two approach in combining TACE and sorafenib: 1) concurrent treatment and 2) sequential treatment. The concurrent TACE and sorafenib treatment showed increased treatment-related toxicity and moderate clinical benefits but no significant survival benefits. ${ }^{24}$ In order to lower the treatment-related toxicity but to maintain the synergistic effect of TACE and sorafenib treatment, our study focused on the sequential treatment.

Within the sequential treatment strategy, treating locoregional therapy first and the sorafenib thereafter has a biological benefit compared to the opposite sequence. It is because locoregional treatments tend to enhance tumor immunity, and increased tumor immunity increases the systemic therapy's effect. There was a significant survival gain with adjuvant chemokine-induced killer cell treatment in patients who underwent locoregional therapy such as surgery, radiofrequency ablation, or ethanol ablation. ${ }^{25}$ Treating TACE initially in advanced HCC patients is expected to have a similar effect as the TACE can similarly obliterate intrahepatic tumors faster than sorafenib. Decreased tumor volume is related to a decreased population of myeloidderived suppressor cells (MDSCs) and regulatory T-cells $\left(\mathrm{T}_{\text {reg }} \mathrm{s}\right){ }^{26,27}$ This immune-modulating effect increases the chance of sorafenib response afterward. ${ }^{28}$

Patients who were treated sequentially (crossover subgroup) survived significantly longer than patients who were not (no crossover subgroup). Moreover, TACE-first treatment tended to improve OS in advanced HCC patients. To sum up, the TACE-first treatment strategy can be an attractive strategy in treating advanced HCC patients.

Interestingly, within the PS-matched cohort, the TACEfirst treatment showed a significant survival benefit for HCCs with more aggressive features compared with the sorafenib-first treatment. It is known that patients with high AFP or portal vein invasion respond poorly to sorafenib treatment. ${ }^{29}$ On the other hand, poorly differentiated tumors 


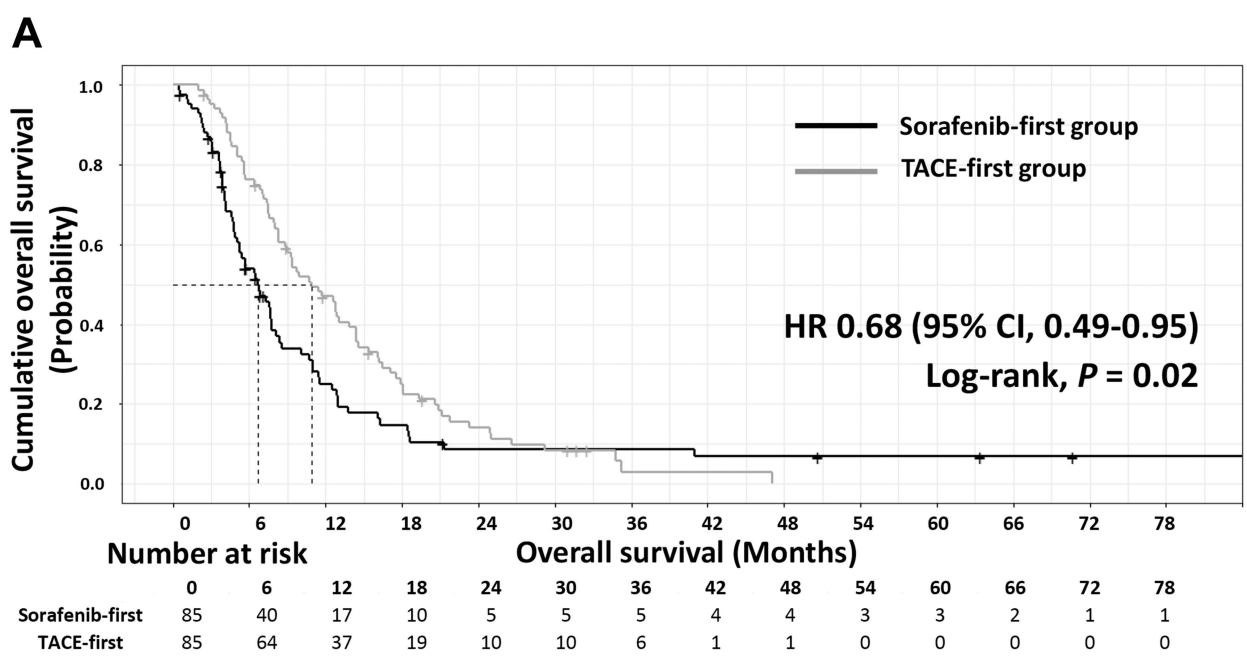

B

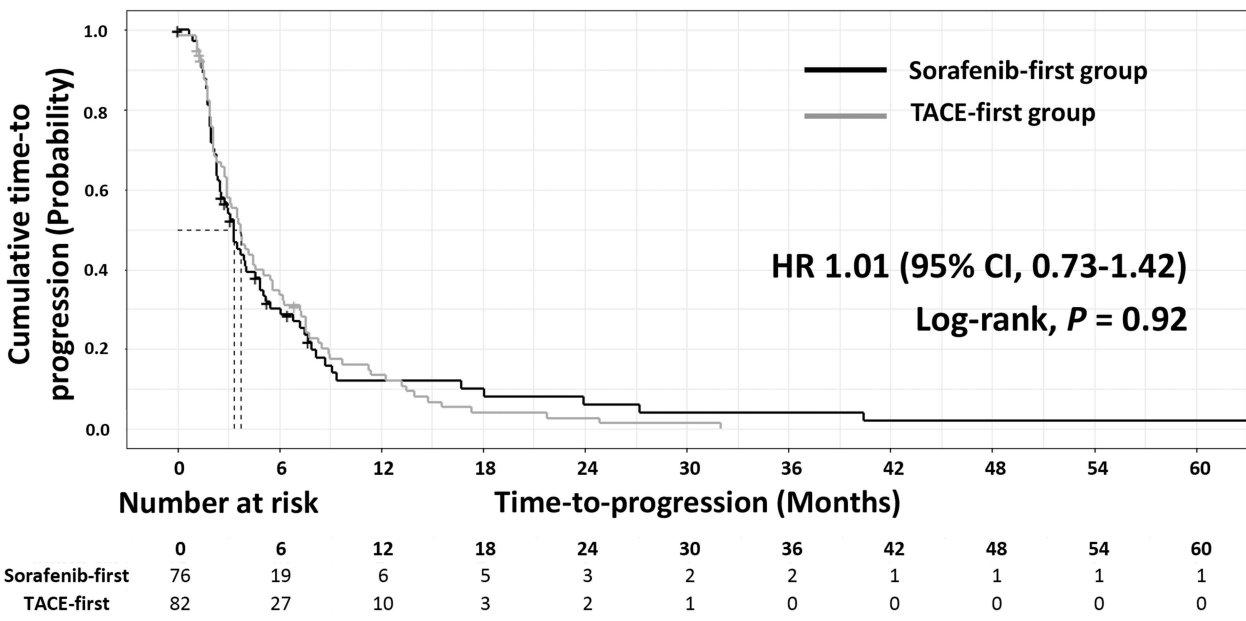

Figure I Cumulative incidence of primary outcomes (PS-matched cohort). (A) Overall survival. (B) Time to progression. Survival curves were compared using the Log rank test. Time to progression was evaluated only in patients with follow-up images.

Abbreviations: $\mathrm{Cl}$, confidence interval; HR, hazard ratio; PS, propensity score; TACE, transarterial chemoembolization.

are more dependent on arterial supply, which makes them more susceptible to TACE treatment. ${ }^{30}$ This makes TACE treatment preferable in aggressive HCC.

Several studies have focused on expanding the indications of TACE treatment and have shown promising results, as mentioned above. ${ }^{15,16}$ It has been confirmed that patients with PVTT or extrahepatic metastasis can be treated safely with TACE. ${ }^{15,16}$ Additionally, as discussed above, in advanced HCC patients with major vessel invasion, locoregional therapy has been shown to confer survival benefits compared with therapy with sorafenib alone. ${ }^{17}$ Given the extension of the indications of TACE and the poor responsiveness to sorafenib in specific groups of patients, aggressive locoregional tumor control with
TACE can be a better option than sorafenib treatment for advanced HCC with aggressive tumor features.

A recent meta-analysis reported a significant correlation between TTP and OS in patients undergoing systemic treatment for advanced HCC. ${ }^{31}$ In our study, however, there was a discrepancy between TTP and OS: TTP was comparable between the two treatment groups, but OS was significantly longer in the TACE-first group. This discrepancy might have occurred due to the different treatment strategies of TACE and sorafenib. In previously TACEtreated patients, a newly developed intrahepatic lesion can be re-treated with additional TACE. On the other hand, there is a limited effect of further sorafenib treatment in progressed patients. Different trends in TTP and OS 
Table 3 Best Overall Response ${ }^{\dagger}$ Between Treatment Groups (PS-Matched Cohort)

\begin{tabular}{|l|l|l|l|}
\hline & $\begin{array}{l}\text { Sorafenib- } \\
\text { First }\end{array}$ & $\begin{array}{l}\text { TACE- } \\
\text { First }\end{array}$ & P-value \\
\hline Complete response & $\mathrm{I}(1.3 \%)$ & $3(3.7 \%)$ & \\
Partial response & $10(13.2 \%)$ & $21(25.6 \%)$ & \\
Stable disease & $25(33.3 \%)$ & $33(40.2 \%)$ & \\
Progressive disease & $39(52.0 \%)$ & $25(30.5 \%)$ & \\
Objective response & $11(14.7 \%)$ & $24(29.3 \%)$ & 0.03 \\
rate $^{\ddagger}$ & & & \\
\hline
\end{tabular}

Notes: Data are expressed as $\mathrm{N}(\%)$. 'Best overall response was evaluated only in patients with a follow-up image. The number of evaluated patients was 75 in the sorafenib-first group and 82 in the TACE-first group. ${ }^{\ddagger}$ Objective response rate $=$ Complete response + Partial response.

Abbreviations: PS, propensity score; TACE, transarterial chemoembolization.

between patients who managed or failed to show objective responses within the PS-matched cohort support this explanation.

One impressive result in this study was in patients with bone metastasis; patients initially treated with sorafenib tended to have a survival benefit compared with the
TACE-first group within the PS-matched cohort. Currently, there is no evidence for sorafenib that supports survival benefits in patients with bone metastasis. ${ }^{29}$ This might be because only a small number of patients with bone metastasis were enrolled. On the other hand, many studies have shown that there is a difference in tumor cell expression according to the site of metastasis. ${ }^{32}$ Further studies comparing treatment responses according to the site of metastasis might be needed.

There are several limitations to our study. As a retrospective analysis of prospectively established cohort study, there is always a risk of imbalance of baseline characteristics between the sorafenib-first and the TACE-first groups. To overcome such bias, PS matching and multivariable analysis were used to calibrate biased patient groups. But still, in retrospective analysis, potential imbalance is at risk, further randomized control trial will be needed for validation. Second, this study was conducted at a single tertiary center where super-selective TACE can be performed by highly experienced experts $(>2500$ cases per year). TACE treatment has inherent operator
A
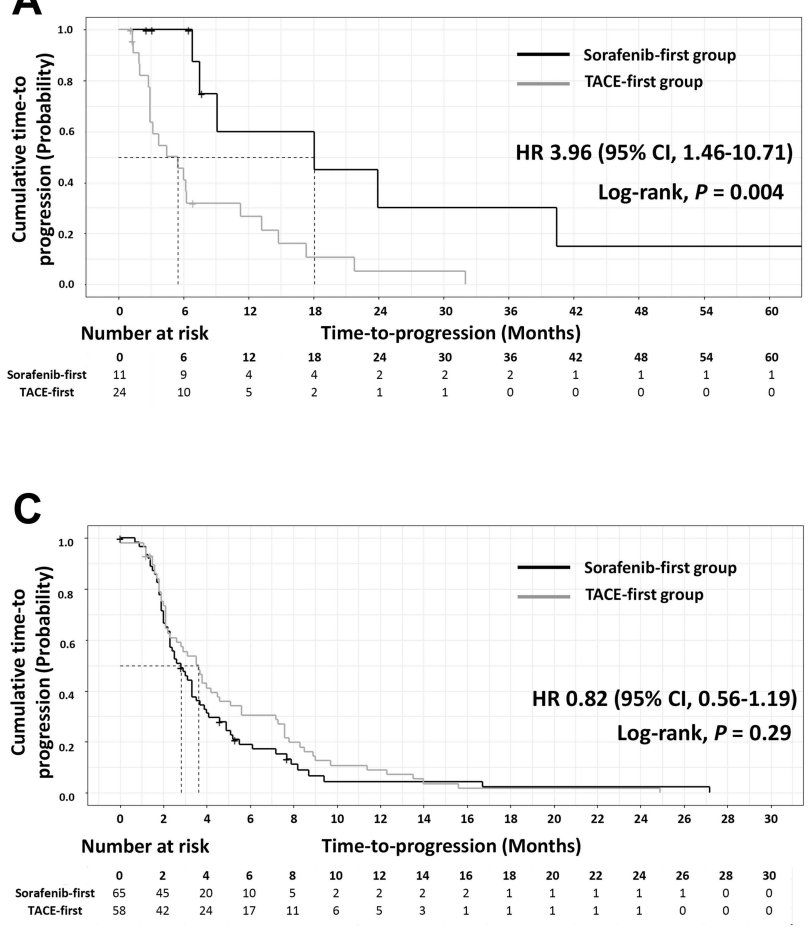

B

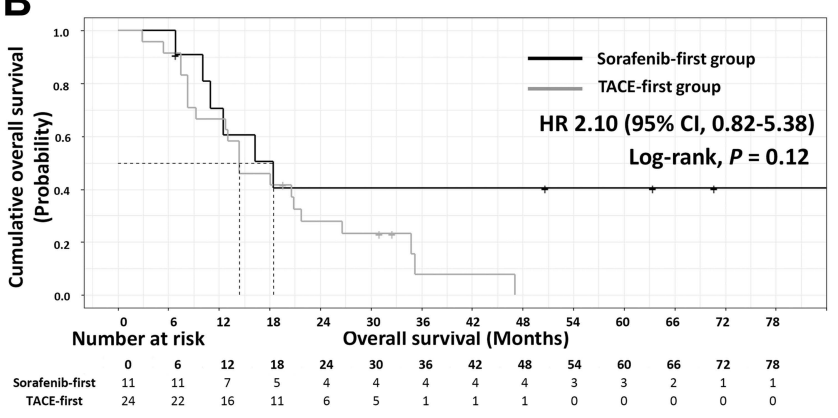

D

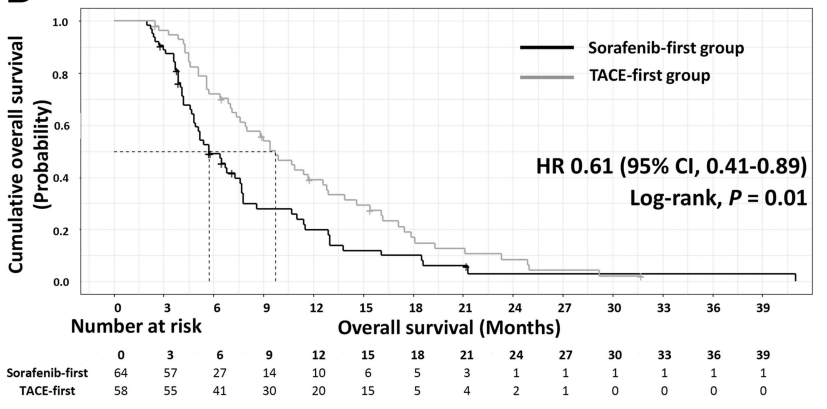

Figure 2 Cumulative incidence of overall survival and time to progression in patients with different responses (PS-matched cohort). (A) Overall survival among patients with objective response (complete or partial response). (B) Time to progression among patients with objective response (complete or partial response). (C) Overall survival among patients without objective response (stable disease or progressive disease). (D) Time to progression among patients without objective response (stable disease or progressive disease). Tumor response was defined in accordance with best overall response. Time to progression was evaluated only in patients with follow-up images. Survival curves were compared using the Log rank test. Time to progression was evaluated only in patients with follow-up images.

Abbreviations: $\mathrm{Cl}$, confidence interval; HR, hazard ratio; PS, propensity score; TACE, transarterial chemoembolization. 
A

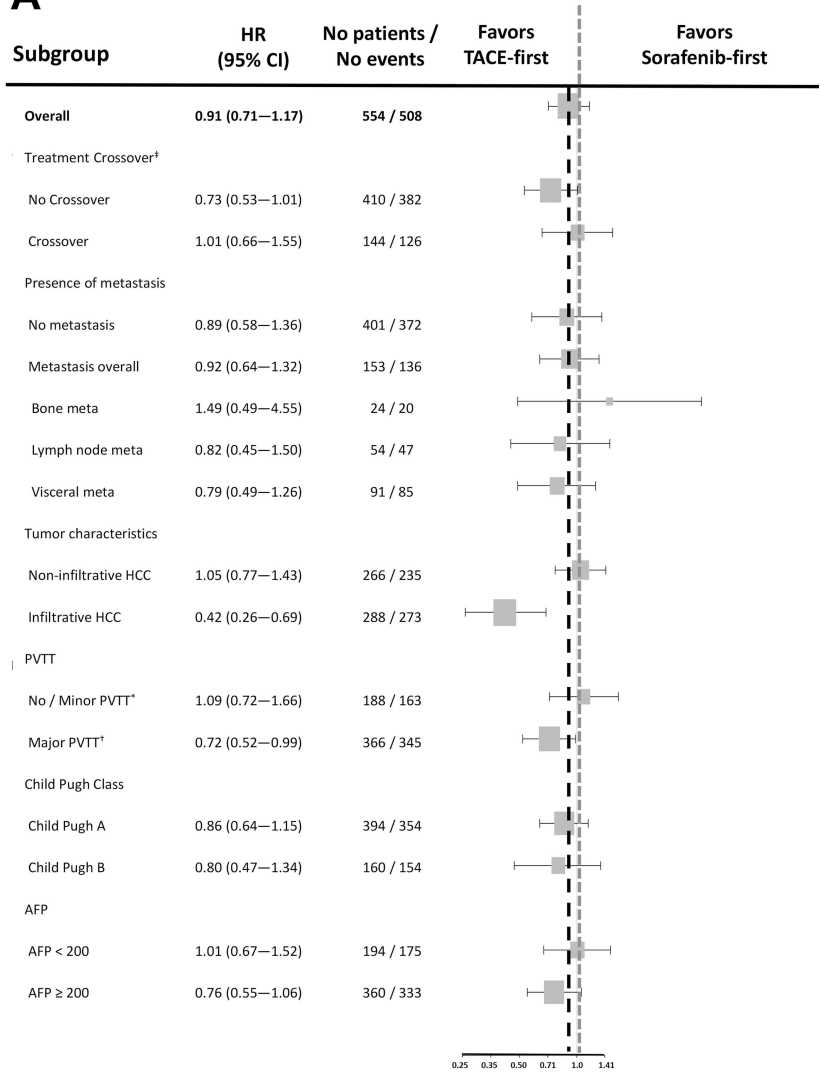

B

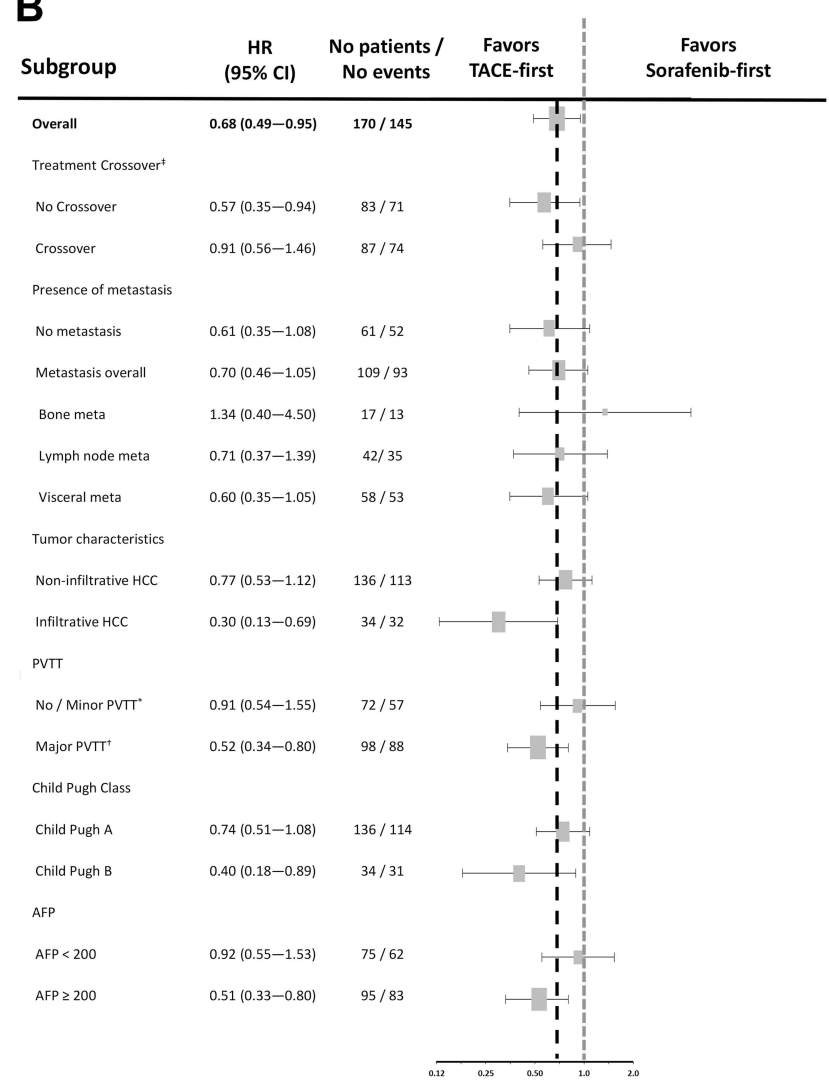

Figure 3 Forest plots of overall survival in patient subgroups. (A) Subgroup analysis of the entire cohort. (B) Subgroup analysis of the PS-matched cohort. Horizontal lines represent $95 \%$ confidence intervals. Confidence intervals and hazard ratios were computed using the Cox proportional hazard model. ${ }^{\ddagger}$ Treatment crossover patients were defined as patients who changed their treatment (TACE to sorafenib or vice versa) throughout the follow-up period. PVTT was classified in accordance with the classification system of the Liver Cancer Study Group of Japan. *Minor PVTT was defined as tumor involvement distal to, but not in, the second-order branches of the portal vein (VpI) or the second-order branches of the portal vein ( $\left.\mathrm{V}_{\mathrm{p} 2}\right)$. 'Major PVTT was defined as tumor involvement of the right or left portal vein $\left(\mathrm{V}_{\mathrm{p}} 3\right)$ or the main portal vein $\left(\mathrm{V}_{\mathrm{p}} 4\right)$. Abbreviations: AFP, alpha-fetoprotein; Cl, confidence interval; HCC, hepatocellular carcinoma; HR, hazard ratio; PS, propensity score; PVTT, portal vein tumor thrombosis; TACE, transarterial chemoembolization.

dependence. ${ }^{33}$ Thus, at centers where staff have less experience with TACE, initial treatment with sorafenib might be a safer option. For further validation of our findings, a multicenter prospective trial is warranted. Third, this study did not include patients treated with other tyrosine kinase inhibitors or immune checkpoint inhibitors such as lenvatinib ${ }^{34}$ regorafenib, ${ }^{7}$ cabozantinib,${ }^{8}$ atezolizumab plus bevacizumab, ${ }^{35}$ or nivolumab, ${ }^{36}$ which are currently used in patients with advanced HCC. Lenvatinib has demonstrated non-inferiority compared with sorafenib and is also recommended as first-line treatment by both the American Association for the Study of Liver Diseases and the European Association for the Study of the Liver. ${ }^{34}$ Atezolizumab plus bevacizumab therapy has shown superior survival benefit compared with sorafenib therapy. ${ }^{35}$ Also, second-line therapy such as regorafenib, ramucirumab, nivolumab, or pembrolizumab is currently available, which might narrow the TACE's role. On the contrary,
TACE was related to decrease immune suppressor cells and promote tumor antigen presentation, which might increase the role in advanced HCC. ${ }^{37,38}$ Additional clinical data needs to be accumulated to establish TACE's role in advanced $\mathrm{HCC}$ in the immune therapy era, but it is quite promising.

In conclusion, in patients with advanced HCC, TACEfirst treatment confers significantly longer OS than sorafenib-first treatment, particularly in advanced HCC with aggressive features. Further randomized control trials with large sample sizes are warranted to validate our findings further.

\section{Acknowledgments}

This work was supported by grants from the National Research Foundation of Korea (NRF) funded by the Korean government (MSIP) (No. 2019R1A2C2010311), from the Seoul National University Hospital Research 
Fund (03-2016-0380), and from the Liver Research Foundation of Korea as part of the Bio Future Strategies Research Project.

\section{Author Contributions}

All authors made a significant contribution to the work reported, whether that is in the conception, study design, execution, acquisition of data, analysis and interpretation, or in all these areas; took part in drafting, revising or critically reviewing the article; gave final approval of the version to be published; have agreed on the journal to which the articles has been submitted; and agree to be accountable for all aspects of the work.

\section{Funding}

Funding sources did not involve in study design, in the collection, analysis and interpretation of data, in the writing of the report, and in the decision to submit the article for publication. The corresponding authors had full access to all study data and had final responsibility for the decision to submit for publication.

\section{Disclosure}

Dr. Lee YB reports receiving research grant from Samjin Pharmaceuticals; Dr. Yu SJ reports lecture fee from Bayer HealthCare Pharmaceuticals; Dr. Kim YJ reports receiving research grants from Bristol-Myers Squibb, Roche, JW Creagene, Bukwang Pharmaceuticals, Handok Pharmaceuticals, Hanmi Pharmaceuticals, Yuhan Pharmaceuticals, Samjin Pharmaceuticals, AstraZeneca, and Pharmaking, and lecture fees from Bayer HealthCare Pharmaceuticals, Gilead Science, MSD Korea, Yuhan Pharmaceuticals, Samil Pharmaceuticals, CJ Pharmaceuticals, Bukwang Pharmaceuticals, and Handok Pharmaceuticals; Dr. Chung JW reports receiving educational grant from Guerbet; Dr. Yoon JH reports receiving research grants from AstraZeneca, Bayer HealthCare Pharmaceuticals, Daewoong Pharmaceuticals, and Bukwang Pharmaceuticals; Dr. Lee JH reports receiving lecture fee from GreenCross Cell, Daewoong Pharmaceuticals, and Gilead Korea. No other potential conflicts of interest relevant to this article were reported.

\section{References}

1. Torre LA, Bray F, Siegel RL, Ferlay J, Lortet-Tieulent J, Jemal A. Global cancer statistics, 2012. CA Cancer J Clin. 2015;65(2):87-108. doi:10.3322/caac. 21262
2. Park JW, Chen M, Colombo M, et al. Global patterns of hepatocellular carcinoma management from diagnosis to death: the BRIDGE Study. Liver Int. 2015;35(9):2155-2166. doi:10.1111/liv.12818

3. European Association for the Study of the Liver. EASL-EORTC clinical practice guidelines: management of hepatocellular carcinoma. J Hepatol. 2012;56(4):908-943.

4. Heimbach JK, Kulik LM, Finn RS, et al. AASLD guidelines for the treatment of hepatocellular carcinoma. Hepatology. 2018;67(1):358380. doi:10.1002/hep.29086

5. Llovet JM, Ricci S, Mazzaferro V, et al. Sorafenib in advanced hepatocellular carcinoma. $N$ Engl J Med. 2008;359(4):378-390. doi:10.1056/NEJMoa0708857

6. Cheng AL, Kang YK, Chen Z, et al. Efficacy and safety of sorafenib in patients in the Asia-Pacific region with advanced hepatocellular carcinoma: a Phase III randomised, double-blind, placebo-controlled trial. Lancet Oncol. 2009;10(1):25-34. doi:10.1016/S1470-2045(08) 70285-7

7. Bruix J, Qin S, Merle P, et al. Regorafenib for patients with hepatocellular carcinoma who progressed on sorafenib treatment (RESORCE): a randomised, double-blind, placebo-controlled, Phase 3 trial. Lancet. 2017;389(10064):56-66. doi:10.1016/S0140-6736(16)32453-9

8. Abou-Alfa GK, Meyer T, Cheng AL, et al. Cabozantinib in patients with advanced and progressing hepatocellular carcinoma. $N$ Engl J Med. 2018;379(1):54-63. doi:10.1056/NEJMoa1717002

9. Zhu AX, Kang YK, Yen CJ, et al. Ramucirumab after sorafenib in patients with advanced hepatocellular carcinoma and increased $\alpha$ fetoprotein concentrations (REACH-2): a randomised, double-blind, placebo-controlled, phase 3 trial. Lancet Oncol. 2019;20(2):282-296. doi:10.1016/S1470-2045(18)30937-9

10. US Food and Drug Administration. FDA Grants Accelerated Approval to Nivolumab for HCC Previously Treated with Sorafenib; 2017. 2018.

11. US Food and Drug Administration. FDA Grants Accelerated Approval to Pembrolizumab for Hepatocellular Carcinoma. 2018.

12. Uka K, Aikata H, Takaki S, et al. Clinical features and prognosis of patients with extrahepatic metastases from hepatocellular carcinoma. World J Gastroenterol. 2007;13(3):414 420. doi:10.3748/wjg.v13.i3.414

13. Jung SM, Jang JW, You CR, et al. Role of intrahepatic tumor control in the prognosis of patients with hepatocellular carcinoma and extrahepatic metastases. J Gastroenterol Hepatol. 2012;27(4):684-689. doi:10.1111/j.1440-1746.2011.06917.x

14. Galle PR, Forner A, Llovet JM, et al. EASL clinical practice guidelines: management of hepatocellular carcinoma. J Hepatol. 2018;69 (1):182-236.

15. Yoo DJ, Kim KM, Jin YJ, et al. Clinical outcome of 251 patients with extrahepatic metastasis at initial diagnosis of hepatocellular carcinoma: does transarterial chemoembolization improve survival in these patients? J Gastroenterol Hepatol. 2011;26(1):145-154. doi:10.1111/j.1440-1746.2010.06341.x

16. Chung GE, Lee JH, Kim HY, et al. Transarterial chemoembolization can be safely performed in patients with hepatocellular carcinoma invading the main portal vein and may improve the overall survival. Radiology. 2011;258(2):627-634. doi:10.1148/radiol.10101058

17. Yoon SM, Ryoo BY, Lee SJ, et al. Efficacy and safety of transarterial chemoembolization plus external beam radiotherapy vs sorafenib in hepatocellular carcinoma with macroscopic vascular invasion: a randomized clinical trial. JAMA Oncol. 2018;4(5):661-669. doi:10.1001/jamaoncol.2017.5847

18. Kudo M, Kitano M, Sakurai T, Nishida N. General rules for the clinical and pathological study of primary liver cancer, nationwide follow-up survey and clinical practice guidelines: the outstanding achievements of the liver cancer study group of Japan. Dig Dis. 2015;33(6):765-770. doi:10.1159/000439101

19. Kim HC, Miyayama S, Chung JW. Selective chemoembolization of caudate lobe hepatocellular carcinoma: anatomy and procedural techniques. Radiographics. 2019;39(1):289-302. doi:10.1148/ rg.2019180110 
20. Choi JW, Kim HC, Lee JH, et al. Transarterial chemoembolization of hepatocellular carcinoma with segmental portal vein tumour thrombus. Eur Radiol. 2017;27(4):1448-1458. doi:10.1007/s00330-016-4511-3

21. Lencioni R, Llovet JM. Modified RECIST (mRECIST) assessment for hepatocellular carcinoma. Semin Liver Dis. 2010;30(1):52-60. doi:10.1055/s-0030-1247132

22. Kudo M, Matsui O, Izumi N, et al. Transarterial chemoembolization failure/refractoriness: JSH-LCSGJ criteria 2014 update. Oncology. 2014;87(Suppl 1):22-31. doi:10.1159/000368142

23. Garnett SA, Martin M, Jerusalem G, et al. Comparing duration of response and duration of clinical benefit between fulvestrant treatment groups in the CONFIRM trial: application of new methodology. Breast Cancer Res Treat. 2013;138(1):149-155. doi:10.1007/s10549012-2395-8

24. Park JW, Kim YJ, Kim DY, et al. Sorafenib with or without concurrent transarterial chemoembolization in patients with advanced hepatocellular carcinoma: the phase III STAH trial. J Hepatol. 2019;70(4):684-691. doi:10.1016/j.jhep.2018.11.029

25. Lee JH, Lee JH, Lim YS, et al. Adjuvant immunotherapy with autologous cytokine-induced killer cells for hepatocellular carcinoma. Gastroenterology. 2015;148(7):1383-1391.e1386. doi:10. 1053/j.gastro.2015.02.055

26. Beyer M, Schultze JL. Regulatory T cells in cancer. Blood. 2006;108 (3):804-811. doi:10.1182/blood-2006-02-002774

27. Mizukoshi E, Yamashita T, Arai K, et al. Myeloid-derived suppressor cells correlate with patient outcomes in hepatic arterial infusion chemotherapy for hepatocellular carcinoma. Cancer Immunol Immunother. 2016;65(6):715-725. doi:10.1007/s00262-016-1837-2

28. Chang CJ, Yang YH, Chiu CJ, et al. Targeting tumor-infiltrating Ly6G(+) myeloid cells improves sorafenib efficacy in mouse orthotopic hepatocellular carcinoma. Int $J$ Cancer. 2018;142 (9):1878-1889. doi:10.1002/ijc.31216

29. Bruix J, Cheng A-L, Meinhardt G, Nakajima K, De Sanctis Y, Llovet J. Prognostic factors and predictors of sorafenib benefit in patients with hepatocellular carcinoma: analysis of two phase III studies. J Hepatol. 2017;67(5):999-1008. doi:10.1016/j.jhep.2017.06.026

30. Matsui O, Kadoya M, Kameyama T, et al. Benign and malignant nodules in cirrhotic livers: distinction based on blood supply. Radiology. 1991;178(2):493-497. doi:10.1148/radiology.178.2.1846 240
31. Lee DW, Jang MJ, Lee KH, et al. TTP as a surrogate endpoint in advanced hepatocellular carcinoma treated with molecular targeted therapy: meta-analysis of randomised controlled trials. $\mathrm{Br} J$ Cancer. 2016;115(10):1201-1205. doi:10.1038/bjc.2016.322

32. Eccles SA, Welch DR. Metastasis: recent discoveries and novel treatment strategies. Lancet. 2007;369(9574):1742-1757. doi:10.10 16/S0140-6736(07)60781-8

33. Facciorusso A, Licinio R, Muscatiello N, Di Leo A, Barone M. Transarterial chemoembolization: evidences from the literature and applications in hepatocellular carcinoma patients. World J Hepatol. 2015;7(16):2009-2019. doi:10.4254/wjh.v7.i16.2009

34. Kudo M, Finn RS, Qin S, et al. Lenvatinib versus sorafenib in first-line treatment of patients with unresectable hepatocellular carcinoma: a randomised phase 3 non-inferiority trial. Lancet. 2018;391(10126):1163-1173. doi:10.1016/S0140-6736 (18)30207-1

35. Finn RS, Qin S, Ikeda M, et al. Atezolizumab plus bevacizumab in unresectable hepatocellular carcinoma. $N$ Engl J Med. 2020;382 (20):1894-1905. doi:10.1056/NEJMoa1915745

36. El-Khoueiry AB, Sangro B, Yau T, et al. Nivolumab in patients with advanced hepatocellular carcinoma (CheckMate 040): an open-label, non-comparative, Phase 1/2 dose escalation and expansion trial. Lancet. 2017;389(10088):2492-2502. doi:10.1016/S0140-6736(17) 31046-2

37. Liao J, Xiao J, Zhou Y, Liu Z, Wang C. Effect of transcatheter arterial chemoembolization on cellular immune function and regulatory $\mathrm{T}$ cells in patients with hepatocellular carcinoma. Mol Med Rep. 2015;12(4):6065-6071. doi:10.3892/mmr.2015.4171

38. Singh P, Toom S, Avula A, Kumar V, Rahma OE. The immune modulation effect of locoregional therapies and its potential synergy with immunotherapy in hepatocellular carcinoma. $J$ Hepatocell Carcinoma. 2020;7:11-17. doi:10.2147/JHC.S187121
Journal of Hepatocellular Carcinoma

\section{Publish your work in this journal}

The Journal of Hepatocellular Carcinoma is an international, peerreviewed, open access journal that offers a platform for the dissemination and study of clinical, translational and basic research findings in this rapidly developing field. Development in areas including, but not limited to, epidemiology, vaccination, hepatitis therapy, pathology and molecular tumor classification and prognostication are al considered for publication. The manuscript management system is completely online and includes a very quick and fair peer-review system, which is all easy to use. Visit http://www.dovepress.com/ testimonials.php to read real quotes from published authors. 